\title{
Chronic airflow obstruction after successful treatment of multidrug-resistant tuberculosis
}

\author{
Anthony L. Byrne ${ }^{1,2,3}$, Ben J. Marais ${ }^{1,3,4}$, Carole D. Mitnick ${ }^{2,5}$ \\ Frances L. Garden ${ }^{6,7,8}$, Leonid Lecca 2,5 , Carmen Contreras' ${ }^{2}$ 'Yaninna Yauri', \\ Fanny Garcia $^{2}$ and Guy B. Marks $1,3,6,7,8$
}

Affiliations: ${ }^{1}$ Department of Medicine, Central Clinical School, University of Sydney, Sydney, Australia. ${ }^{2}$ Socios En Salud Sucursal Perú, Partners In Health, Lima, Peru. ${ }^{3}$ Centre for Research Excellence in Tuberculosis (TB-CRE), Sydney, Australia. ${ }^{4}$ Marie Bashir Institute for Infectious Diseases and Biosecurity, University of Sydney, Sydney, Australia. ${ }^{5}$ Harvard Medical School, Boston, MA, USA. ${ }^{6}$ The Woolcock Institute of Medical Research, Sydney, Australia. ${ }^{7}$ South Western Sydney Clinical School, University of New South Wales, Sydney, Australia. ${ }^{8}$ Ingham Institute of Applied Medical Research, Sydney, Australia. ${ }^{9}$ Ministry of Health, Red de Salud Lima Ciudad, Lima, Peru.

Correspondence: Anthony Byrne, St Vincent's Hospital Sydney, Heart Lung Clinic, Xavier Building, 390 Victoria Street 2010, Darlinghurst, NSW 2010, Australia. E-mail: abyrnelamed.usyd.edu.au

ABSTRACT Cross-sectional studies reveal an association between tuberculosis (TB) and chronic airflow obstruction, but cannot adequately address confounding. We hypothesised that treated pulmonary TB is an independent risk factor for chronic airflow obstruction.

The Pulmones Post TB cohort study enrolled participants from Lima, Peru, aged 10-70 years with a history of drug-susceptible (DS)- or multidrug-resistant (MDR)-TB who had completed treatment and were clinically cured. Unexposed participants without TB were randomly selected from the same districts. We assessed respiratory symptoms, relevant environmental exposures, and spirometric lung function pre- and post-bronchodilator.

In total, 144 participants with DS-TB, 33 with MDR-TB and 161 unexposed participants were fully evaluated. Compared with unexposed participants, MDR-TB patients had lower lung volumes (adjusted mean difference in forced vital capacity $-370 \mathrm{~mL}, 95 \% \mathrm{CI}-644--97)$ and post-bronchodilator airflow obstruction (adjusted OR 4.89, 95\% CI 1.27-18.78). Participants who had recovered from DS-TB did not have lower lung volumes than unexposed participants, but were more likely to have a reduced forced expiratory volume in $1 \mathrm{~s}$ /forced vital capacity ratio $<0.70$ (adjusted OR 2.47, 95\% CI 1.01-6.03).

Individuals successfully treated for TB may experience long-lasting sequelae. Interventions facilitating earlier TB treatment and management of chronic respiratory disease should be explored.

@ERSpublications

Treated MDR-TB results in loss of lung volume; MDR-TB and susceptible TB can result in chronic airflow obstruction http://ow.ly/yM9H30bRDha

Cite this article as: Byrne AL, Marais BJ, Mitnick CD, et al. Chronic airflow obstruction after successful treatment of multidrug-resistant tuberculosis. ERJ Open Res 2017; 3: 00026-2017 [https://doi.org/10.1183/23120541.00026-2017].

This article has supplementary material available from openres.ersjournals.com

Received: Feb 272017 | Accepted after revision: May 142017

Support statement: This study was supported by the National Health and Medical Research Council Centre of Research Excellence, the University of Sydney, and the Woolcock Institute of Medical Research. The funding sources were external to the study design and interpretation of results. Funding information for this article has been deposited with the Crossref Funder Registry.

Conflict of interest: None declared

Copyright $\odot$ ERS 2017. This article is open access and distributed under the terms of the Creative Commons Attribution Non-Commercial Licence 4.0. 


\section{Introduction}

Chronic obstructive pulmonary disease (COPD) results in 2.8 million deaths each year (291 per 100 000) and is the third leading cause of mortality worldwide [1]. Although COPD is commonly thought of as an older person's disease associated with cigarette smoke exposure, there is increased awareness of non-smoking-related risk factors that also contribute to its development $[2,3]$. The link between cigarette smoking and COPD was first characterised in high-income countries, but it is rarely appreciated that, globally, most people with COPD live in low- and middle-income countries where cigarette smoking is one of many risk factors and younger people are frequently affected $[4,5]$. Other factors such as indoor use of bio-combustible fuel, outdoor air pollution and respiratory infections have been more strongly associated with chronic respiratory disease in young people than smoking in some settings [6, 7].

Tuberculosis (TB) is a common respiratory infection that typically affects young adults in low- and middle-income countries [8]. It is estimated that 10.4 million people developed TB in 2015, resulting in 1.4 million deaths (281 per 100000) [8]. Successful treatment requires multiple antibiotics over several months; with good adherence, cure rates are generally excellent. However, the increasing incidence of multidrug-resistant (MDR)-TB in some countries is a cause for concern and threatens to reverse recent gains in global TB control [9]. Since sputum smear microscopy cannot differentiate drug-susceptible (DS)-TB from drug-resistant (DR)-TB, many patients with DR-TB experience prolonged diagnostic delay and potentially more lung tissue destruction [10].

TB may be a major contributor to the prevalence of chronic airflow obstruction in TB endemic areas [11-14], especially in younger age groups. Several case series and cross-sectional studies and a single cohort study [15], in which the presence of COPD was inferred from data on medication prescriptions, have demonstrated an association between the presence of chronic airflow obstruction and previous TB [14]. However, these studies may be subject to misclassification of exposure or end-point, which limits their utility. Furthermore, it is possible that the detrimental effects observed in these studies relate to an era when diagnostic delay was more prolonged and the treatment of DS-TB less effective than it is now. These findings may not be applicable to patients diagnosed today and treated with modern quality-assured regimens [16]. In contrast, patients with DR-TB, for whom treatment is often delayed and is less effective, may still be at risk of adverse respiratory outcomes. Hence, there is a need for well-designed cohort studies, in which recall bias can be eliminated and selection bias minimised, to separately evaluate the lung function consequences of DS- and DR-TB.

In this study, we sought to determine if successfully treated pulmonary TB (both DS- and MDR-TB) results in adverse respiratory outcomes, including impaired lung function, respiratory symptoms and airway inflammation, independent of other risk factors such as cigarette smoke or indoor air pollution exposure. The primary objective of this study was to determine if successfully treated pulmonary $\mathrm{TB}$ (laboratory-confirmed DS or MDR Mycobacterium tuberculosis) is an independent risk factor for impaired lung function, specifically chronic airflow limitation as defined by post-bronchodilator spirometry. Secondary objectives were to characterise the respiratory symptoms, measured airway inflammation and functional capacity of successfully treated pulmonary TB patients compared with participants with no prior TB disease.

\section{Materials and methods}

Study design and setting

The Pulmones Post TB study (study protocol OEE-040-14) was a population-based historical cohort study performed in Lima, Peru, as per the STROBE statement [17]. The study was conducted in two poor and highly urbanised communities (La Victoria and Cercado de Lima (central Lima District); supplementary appendix S1.1) located in central Lima with a population of around 500000 people (figure 1). The sample size calculation is detailed in supplementary appendix S1.2.

\section{Participants and selection criteria}

Study participants were current residents aged between 10 and 70 years, who had successfully completed treatment for pulmonary DS- or MDR-TB. This TB (exposed) cohort included a randomly selected sample of eligible patients who had (successfully) completed DS-TB treatment in the 12 months prior to November 1, 2014, and all eligible patients who had successfully completed MDR-TB treatment in the 36 months prior to that date. Treatment of MDR-TB in Peru is guided and supervised by a panel of expert pulmonologists, with monthly sputum samples assessed for acid-fast bacilli (AFB) smear positivity. All study participants treated for MDR-TB had confirmed clinical cure. Patients with symptoms (or chest radiographs) suggestive of active TB were referred to the local health centre for sputum assessment and clinical review. We also collected sputum samples for AFB microscopy and culture in all patients previously treated for MDR-TB, regardless of symptoms or chest radiograph findings. None returned 


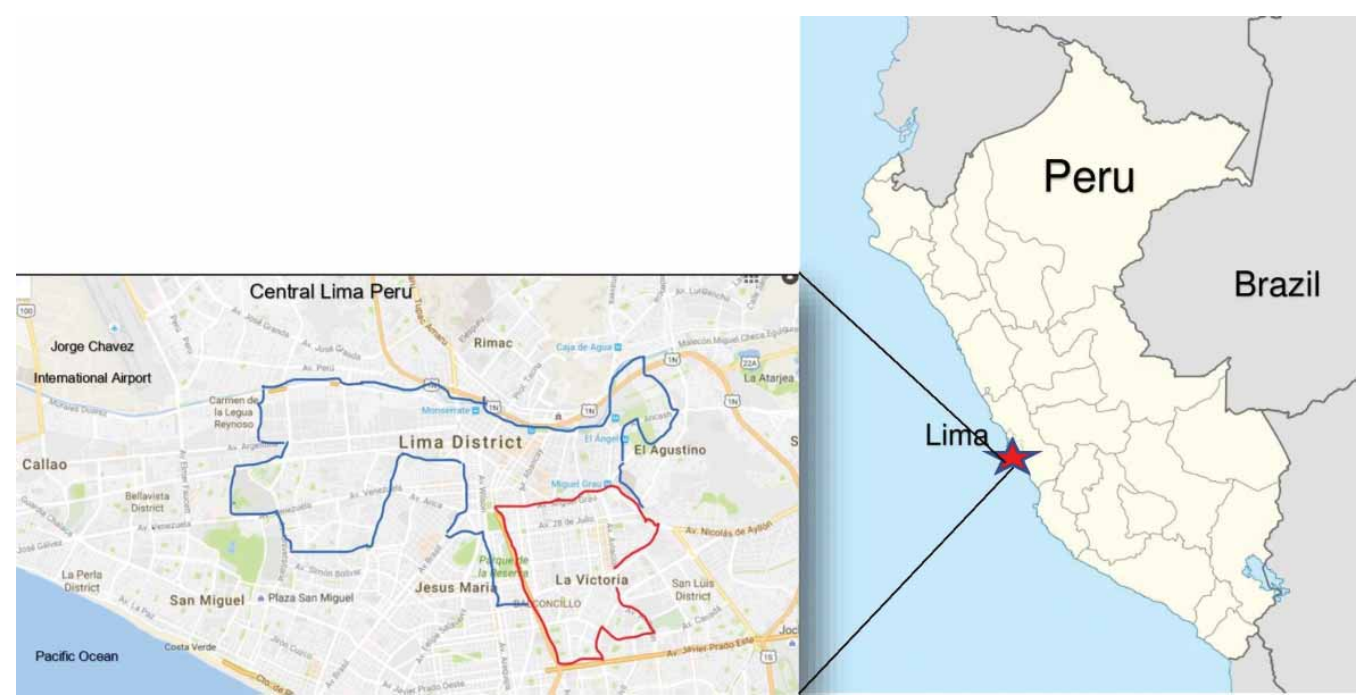

FIGURE 1 Study locations of central Lima, Peru: districts of La Victoria (red) and Cercado de Lima (central Lima District) (blue). Map data: (C2017 Google.

positive cultures for M. tuberculosis (supplementary appendix S1.3). People who had never had TB were selected from the same geographical area using a "random walk" sampling technique (supplementary appendix S1.4) [18].

\section{Study procedures}

All participants completed a standardised questionnaire, anthropometric measurements, a clinical assessment, exhaled nitric oxide fraction $\left(\mathrm{FeNO}_{\mathrm{e}}\right)$ breath analysis, skin prick testing, a 6-min walk distance (6MWD) test, and spirometric lung function testing performed pre- and post-bronchodilator (supplementary appendix S1.5). The questions were based on those used for the BOLD and PLATINO studies $[4,5]$, and included assessments of respiratory symptoms such as cough, wheeze and dyspnoea, as well as additional TB-specific questions and potential confounders (current or former smoking, exposure to environmental tobacco smoke, bio-combustible fuel and occupational dust).

\section{Primary and secondary end-points}

The primary end-point was defined as a post-bronchodilator forced expiratory volume in $1 \mathrm{~s}\left(\mathrm{FEV}_{1}\right) /$ forced vital capacity (FVC) ratio $<0.70$ and/or $\mathrm{FEV} 1 / \mathrm{FVC}<2 \mathrm{sD}$ below the mean of the reference population. The secondary end-points were respiratory symptoms of cough (with or without phlegm), dyspnoea or wheeze, airway inflammation as measured by $F$ eNO ( ppb) and functional capacity determined by 6 MWD $(\mathrm{m})$. See supplementary appendix S1.5 for more details.

\section{Statistical analysis}

The best post-bronchodilator FEV1 and the best post-bronchodilator FVC were identified and used to calculate the FEV1/FVC ratio [19]. The lower limit of normal (LLN) for the FEV1/FVC ratio was calculated using the Global Lung Initiative (GLI) reference values, which standardise for age, height and sex [20]. Internal control values were used to transform observed $\mathrm{FEV} 1, \mathrm{FVC}$ and $\mathrm{FEV} 1 / \mathrm{FVC}$ ratio values to normalised z-scores. Ethnicity categories within the GLI were based on a participant's self-assigned ethnicity (Caucasian, African-American or mixed). In addition, age-, height- and sex-adjusted mean expected values and standard deviations were calculated using data from the unexposed (control group) participants.

The distribution of z-scores for spirometry by study group was plotted using kernel density smoothing plots. Participants were defined as having low values for each of the spirometric end-points if their $\mathrm{Z}$-scores were $<-2.0$ ( $>2 \mathrm{sD}$ below the mean of the control population) [21]. The prevalence of wheeze in the past 12 months, cough for $>3$ months without sputum, cough with sputum for $>3$ months, dyspnoea (as defined by a modified Medical Research Council dyspnoea scale score $\geqslant 2$ ) [22], low FEV1, low FVC and low FEV1/FVC ratio was estimated and compared between groups by the Chi-squared test. The effect of DS- or MDR-TB exposure on reported symptoms and adverse spirometric outcomes was estimated as an odds ratio, with 95\% confidence intervals, using logistic regression. Covariates measured by questionnaire (as listed previously) and skin prick tests were included as potential confounders. We also 
tested the effect of DS- and MDR-TB exposure on the level of spirometric lung function, FeNO and $6 \mathrm{MWD}$, estimated as mean differences, with $95 \%$ confidence intervals, using linear regression and adjusting for the covariates listed above. All statistical analysis was performed using SAS version 9.4 (SAS Institute, Cary, NC, USA) and the kernel density smoothing plots were created using Stata version 14 (StataCorp, College Station, TX, USA).

\section{Ethics}

All study participants, or their legal guardian in the case of adolescents and minors, provided written informed consent. The study was approved by the Comité Institucional de Ética en Investigación, Instituto Nacional de Salud and Socios En Salud Sucursal Perú.

\section{Results}

Figure 2 provides an overview of study enrolment and participation. Of 410 patients treated for DS-TB in the preceding year, $326(80 \%)$ were still alive and resident in the study area. We randomly selected 231 for inclusion in the study sample, and could contact and recruit 144 (62\%). Among 131 patients who had been treated for MDR-TB in the preceding 3 years, 96 (73\%) had successful treatment outcomes, and were still alive and resident in the study area. These former patients were selected for inclusion; finally, 33

La Victoria and Cercado de Lima (central Lima District)
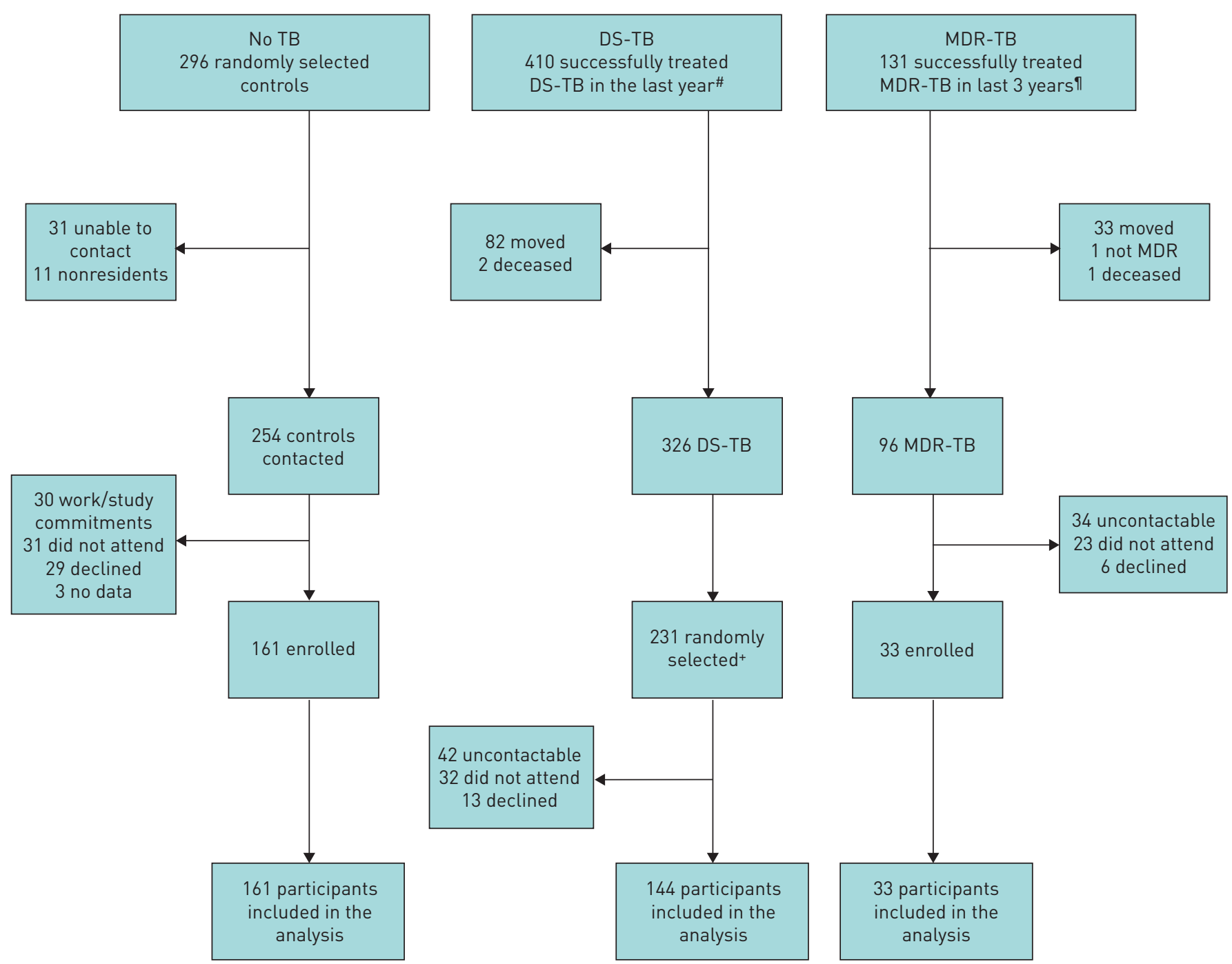

FIGURE 2 Flowchart of study enrolment and participation. DS: drug-susceptible; TB: tuberculosis; MDR: multidrug-resistant. ${ }^{\#}$ : successfully completed treatment for DS-TB between November 1, 2013 and October 31, 2014; ${ }^{9}$ : successfully completed treatment for MDR-TB between November 1, 2011 and October 31,$2014 ;{ }^{+}$: using a computer-generated list of random numbers, corresponding to the de-identified list of DS-TB patients. 
(34\%) were contactable and agreed to participate. Among 296 randomly selected community participants without TB, 161 (54\%) agreed to participate in the study.

Baseline data on sociodemographic and anthropometric characteristics and exposures for all three cohorts are presented in table 1. Participants in the control group cohort were older (median age 38 years) than the participants in the treated TB subcohorts (median 30 years for DS-TB and 29 years for MDR-TB) and included fewer males (30\% compared with $58 \%$ for both DS- and MDR-TB). Among the TB subcohorts, there was no difference in age or the number of participants aged $<18$ years $(6.1 \%)$. The prevalence of current or past cigarette smoking was low for all three groups $(\leqslant 17 \%)$. Exposure to bio-combustible fuel (indoor air pollution) was similar across groups (19\% for MDR-TB; $25 \%$ for both "No TB" and DS-TB).

Table 2 shows the prevalence of reported respiratory symptoms and the functional outcomes measured. We found no significant difference between the three groups in the prevalence of cough (with or without phlegm), wheeze, dyspnoea, or mean FeNO or 6MWD. A high percentage of the MDR-TB subcohort had low FVC (23.3\% compared with $2.9 \%$ among unexposed community controls), while FEV1/FVC $<0.70$ was more prevalent in the DS- and MDR-TB subcohorts (14.0\% and $16.7 \%$, respectively) compared with community controls $(7.1 \%)$.

Table 3 presents univariate and multivariate logistic regression analyses for respiratory outcomes in the DS- and MDR-TB subcohorts compared with community controls, while table 4 shows the absolute differences between these groups. Airflow obstruction was more prevalent in the MDR-TB subcohort than in the unexposed community controls, especially when defined based on FEV1/FVC <LLN (adjusted OR 3.58, 95\% CI 1.32-9.68). Findings were equivocal in the DS-TB subcohort. Airflow obstruction was statistically significantly more common in the DS-TB subcohort compared with the controls only when airflow obstruction was defined as $\mathrm{FEV}_{1}, \mathrm{FVC}<0.70$. Compared with the control cohort, spirometric lung volumes (FEV1 and FVC) were reduced in the MDR- but not the DS-TB subcohort (tables 2 and 3). Neither of the TB subcohorts differed from community controls with respect to FeNO or 6MWD (table 4).

\section{TABLE 1 Demographics and baseline characteristics of all study participants}

\begin{tabular}{|c|c|c|c|}
\hline & No TB & DS-TB & MDR-TB \\
\hline Subjects & 161 & 144 & 33 \\
\hline \multicolumn{4}{|l|}{ Demographics } \\
\hline Age years & $37.6(20.7-52.5)$ & $30.0(20.4-45.2)$ & $29.0(22.4-37.7)$ \\
\hline$<18$ years & $30(18.6)$ & $18(6.1)$ & $2(6.1)$ \\
\hline Male & $49(30.4)$ & $83(57.6)$ & $19(57.6)$ \\
\hline Height cm & $158.1 \pm 8.8$ & $161.1 \pm 9.5$ & $160.9 \pm 11.1$ \\
\hline $\mathrm{BMI} \mathrm{kg} \cdot \mathrm{m}^{-2}$ & $26.0 \pm 5.1$ & $24.5 \pm 4.2$ & $24.0 \pm 3.8$ \\
\hline Born in Lima & $112(69.6)$ & $102(70.8)$ & $28(84.9)$ \\
\hline \multicolumn{4}{|l|}{ Ethnicity } \\
\hline Caucasian & $16 / 152(10.5)$ & $9 / 136(6.6)$ & $1 / 28(3.6)$ \\
\hline African-American & 5/152 (3.3) & $2 / 136(1.5)$ & $0 / 28(0.0)$ \\
\hline Mestizo (mixed) & $131 / 152(86.2)$ & $125 / 136$ (91.9) & $27 / 28(96.4)$ \\
\hline \multicolumn{4}{|l|}{ Socioeconomic } \\
\hline Employment & $75 / 160$ (46.9) & $101(70.1)$ & $16 / 32(50.0)$ \\
\hline \multicolumn{4}{|l|}{ Education } \\
\hline Primary or less & 31 (19.3) & $13(9.0)$ & $3(9.1)$ \\
\hline Secondary & 89 (55.3) & 79 (54.9) & $17(51.5)$ \\
\hline Technical & 35 (21.7) & $34(23.6)$ & $5(15.2)$ \\
\hline University & $6(3.7)$ & $18(12.5)$ & 8 (24.2) \\
\hline Crowding Index persons $\cdot \mathrm{m}^{-2}$ & $0.13(0.08-0.23)$ & $0.14(0.08-0.25)$ & $0.13(0.06-0.17)$ \\
\hline \multicolumn{4}{|l|}{ Exposures } \\
\hline Current smoker $\#$ & $17 / 160(10.6)$ & 12 (8.3) & $1 / 32(3.1)$ \\
\hline Ever-smoker & 18/159 (11.3) & $24 / 143(16.8)$ & $4 / 32(12.5)$ \\
\hline Passive smoke & $38 / 160(23.8)$ & $15(10.4)$ & $1(3.0)$ \\
\hline Indoor air pollution? & $39 / 158(24.7)$ & $35 / 143(24.5)$ & $6 / 32(18.8)$ \\
\hline Occupational dust ${ }^{+}$ & $40 / 160(25.0)$ & $54 / 143$ (37.8) & $5(15.2)$ \\
\hline
\end{tabular}

Data are presented as $N$, median (interquartile range), $n(\%)$, mean \pm sD or $n / N(\%)$, unless otherwise stated. TB: tuberculosis; DS: drug-susceptible; MDR: multidrug-resistant; BMI: body mass index. \#: smoked $\geqslant 1$ cigarette in the last 30 days; ${ }^{\text {": }}$ indoor use of bio-combustible fuel such as wood-fire cooking (ever); ${ }^{+}$: worked in a dusty job (ever). 


\begin{tabular}{|c|c|c|c|c|}
\hline & No TB & DS-TB & MDR-TB & p-value \\
\hline Subjects & 161 & 144 & 33 & \\
\hline \multicolumn{5}{|l|}{ Respiratory symptoms } \\
\hline Chronic cough without phlegm ${ }^{\#}$ & $8 / 160(5.0)$ & $11(7.6)$ & 1/32 (3.1) & 0.48 \\
\hline Chronic cough with phlegm ${ }^{\sharp}$ & $15 / 160(9.4)$ & $12(8.3)$ & $4 / 32(12.5)$ & 0.76 \\
\hline Wheeze in the last 12 months & $36 / 160(22.5)$ & $25(17.4)$ & $11 / 32(34.4)$ & 0.09 \\
\hline Dyspnoeaी & $34 / 160$ (21.3) & $25(17.4)$ & $8 / 32(25.0)$ & 0.53 \\
\hline Airway inflammation: FenO ppb & $16.8 \pm 1.9$ & $18.7 \pm 1.8$ & $15.6 \pm 1.7$ & 0.46 \\
\hline $\begin{array}{l}\text { Functional capacity: } 6 \mathrm{MWD} \text { m } \\
\text { Lung function }\end{array}$ & \multicolumn{3}{|c|}{ Lung function } & 0.86 \\
\hline FEV $1 \mathrm{~L}^{+}$ & $2.69 \pm 0.7$ & $2.96 \pm 0.9$ & $2.53 \pm 1.0$ & $<0.01$ \\
\hline Low FEV1 & $7 / 140(5.0)$ & $11 / 129(8.5)$ & $8 / 30(26.7)$ & $<0.01$ \\
\hline $\mathrm{FVC} \mathrm{L}^{+}$ & $3.30 \pm 0.8$ & $3.68 \pm 1.0$ & $3.23 \pm 1.1$ & $<0.01$ \\
\hline Low FVC & $4 / 140$ (2.9) & $6 / 129(4.7)$ & $7 / 30(23.3)$ & $<0.01$ \\
\hline $\mathrm{FEV}_{1} / \mathrm{FVC}^{\S}$ & $0.82 \pm 0.09$ & $0.80 \pm 0.11$ & $0.77 \pm 0.13$ & 0.01 \\
\hline Low FEV $1 / F V C$ & $6 / 140(4.3)$ & $12 / 129$ (9.3) & $5 / 30(16.7)$ & 0.046 \\
\hline $\mathrm{FEV}_{1} / \mathrm{FVC}<0.70^{\S}$ & $10 / 140(7.1)$ & $18 / 129(14.0)$ & $5 / 30(16.7)$ & 0.12 \\
\hline
\end{tabular}

Data are presented as $\mathrm{N}, \mathrm{n} / \mathrm{N}(\%)$ or mean $\pm \mathrm{SD}$, unless otherwise stated. DS: drug-susceptible; MDR: multidrug-resistant; FeNO: fraction of exhaled nitric oxide; 6MWD: 6-min walk distance; FEV1: forced expiratory volume in $1 \mathrm{~s}$; FVC: forced vital capacity. FEV1, FVC and $F E V_{1} / F V C$ all refer to best postbronchodilator values that fulfilled quality control requirements. Low FEV1, low FVC and low FEV1/FVC all refer to <2SD below the mean of the control group. \#: cough (without a cold) most days for at least 3 months each year, with or without phlegm; ${ }^{n}$ : modified Medical Research Council score $\geqslant 2$ [22]; ${ }^{+}$: testing the differences in the mean values of FEV 1 and FVC between the groups was adjusted for age, sex and height; ${ }^{\S}$ : testing the difference in the mean value of $\mathrm{FEV}_{1} / \mathrm{FVC}$ and those with $\mathrm{FEV} 1 / \mathrm{FVC}<0.7$ between the groups was adjusted for age and sex.

Examination of the height-, age- and sex-adjusted density (frequency) plots for FEV1 and FVC (figure 3a and $b$ ) demonstrates that, among the MDR-TB subcohort, most people had a similar distribution of lung volumes to that observed in the community controls (and the DS-TB subcohort). However, there is a substantial subset of this MDR-TB subcohort, seen as a large left-side (lower) tail, with lower than expected lung volumes. In contrast, the distribution of the FEV1/FVC ratio (figure $3 \mathrm{c}$ ) in the MDR-TB subcohort is quite different, with the entire distribution shifted to the left (lower) compared with the distribution for the community controls (and the DS-TB subcohort).

\section{Discussion}

This is the first population-based cohort study to estimate the effect of successfully treated pulmonary TB on lung function and other respiratory health outcomes. We found that despite successful microbiological treatment outcomes, patients treated for MDR-TB had substantially increased risk of impaired lung function and airflow obstruction compared with community controls. In contrast, patients successfully treated for DS-TB had no reduction in spirometric lung volumes compared with people who did not experience $\mathrm{TB}$, but they did have some evidence of airflow obstruction.

The observation that TB causes chronic lung function loss is not new [23]. However, the management of TB has changed a great deal since the first observations were made $>60$ years ago. The introduction of rifampicin and pyrazinamide, together with isoniazid, resulted in dramatically shortened regimens and improved treatment success $[24,25]$. The early bactericidal activity of isoniazid and rifampin, combined with the sterilising ability of rifampin and pyrazinamide, led to cure rates of $>90 \%$ following 6 months of combination therapy [26]. Despite these treatment advances, studies continued to show impaired lung function (both restrictive and obstructive patterns) among previously treated TB patients [11, 12, 14]. However, optimised treatment regimens were not universally implemented before the roll-out of the DOTS (directly observed treatment, short course) strategy in the 1990s and until this time the observed prevalence of functional lung impairment varied widely, depending on the population studied [27-29]. In addition, the interpretation of study findings was complicated by selection bias, recall bias and lack of adjustment for confounders, such as smoking, indoor air pollution, occupational dust exposure and other socioeconomic variables. The present investigation, an historical cohort study in which an unexposed cohort was recruited from the same region that the patient cohorts were derived from and in which a range of important potential confounders were measured, addresses many of the limitations of previous studies. 


\begin{tabular}{|c|c|c|c|c|}
\hline & \multicolumn{2}{|c|}{ DS-TB ${ }^{\#}$} & \multicolumn{2}{|c|}{ MDR-TB ${ }^{\#}$} \\
\hline & OR $(95 \% \mathrm{CI})$ & p-value & OR $(95 \% \mathrm{CI})$ & p-value \\
\hline \multicolumn{5}{|c|}{ Chronic cough without phlegm } \\
\hline Univariate & $1.57(0.61-4.02)$ & 0.35 & $0.61(0.07-5.08)$ & 0.65 \\
\hline Multivariate & $1.34(0.49-3.67)$ & 0.57 & $0.57(0.07-4.90)$ & 0.61 \\
\hline \multicolumn{5}{|c|}{ Chronic cough with phlegm $\pi$} \\
\hline Univariate & $0.88(0.40-1.95)$ & 0.75 & $1.38(0.43-4.47)$ & 0.60 \\
\hline Multivariate & $0.80(0.35-1.84)$ & 0.63 & $1.23(0.37-4.14)$ & 0.74 \\
\hline \multicolumn{5}{|c|}{ Wheeze in last 12 months } \\
\hline Univariate & $0.72(0.41-1.28)$ & 0.27 & $1.80(0.80-4.09)$ & 0.16 \\
\hline Multivariate & $0.70(0.38-1.27)$ & 0.24 & $2.09(0.88-4.99)$ & 0.10 \\
\hline \multicolumn{5}{|l|}{ Dyspnoea $^{+}$} \\
\hline Univariate & $0.78(0.44-1.38)$ & 0.39 & $1.24(0.51-2.99)$ & 0.64 \\
\hline Multivariate & $0.78(0.43-1.43)$ & 0.43 & $1.59(0.63-4.03)$ & 0.33 \\
\hline \multicolumn{5}{|l|}{ Low FEV1 } \\
\hline Univariate & $1.77(0.67-4.72)$ & 0.25 & $6.91(2.28-20.97)$ & $<0.01$ \\
\hline Multivariate & $2.70(0.89-8.17)$ & 0.08 & $7.26(1.97-26.72)$ & $<0.01$ \\
\hline \multicolumn{5}{|l|}{ Low FVC } \\
\hline Univariate & $1.66(0.46-6.02)$ & 0.44 & $10.35(2.81-38.18)$ & $<0.01$ \\
\hline Multivariate & $2.06(0.46-9.29)$ & 0.35 & $8.90(1.88-42.12)$ & $<0.01$ \\
\hline \multicolumn{5}{|l|}{ Low FEV 1 FVC } \\
\hline Univariate & $2.29(0.83-6.30)$ & 0.11 & 4.47 (1.27-15.77) & 0.02 \\
\hline Multivariate & $2.36(0.81-6.87)$ & 0.12 & 4.89 (1.27-18.78) & 0.02 \\
\hline \multicolumn{5}{|l|}{$\mathrm{FEV}_{1} / \mathrm{FVC}<0.70$} \\
\hline Univariate & 2.45 (1.04-5.78) & 0.04 & $3.27(0.98-10.93)$ & 0.06 \\
\hline Multivariate & 2.47 (1.01-6.03) & 0.047 & $3.53(1.00-12.48)$ & 0.05 \\
\hline \multicolumn{5}{|l|}{ FEV $_{1} /$ FVC $<$ LLN } \\
\hline Univariate & $1.68(0.85-3.35)$ & 0.14 & 3.88 (1.54-9.73) & $<0.01$ \\
\hline Multivariate & $1.89(0.91-3.94)$ & 0.09 & 3.58 (1.32-9.68) & 0.01 \\
\hline \multicolumn{5}{|c|}{ 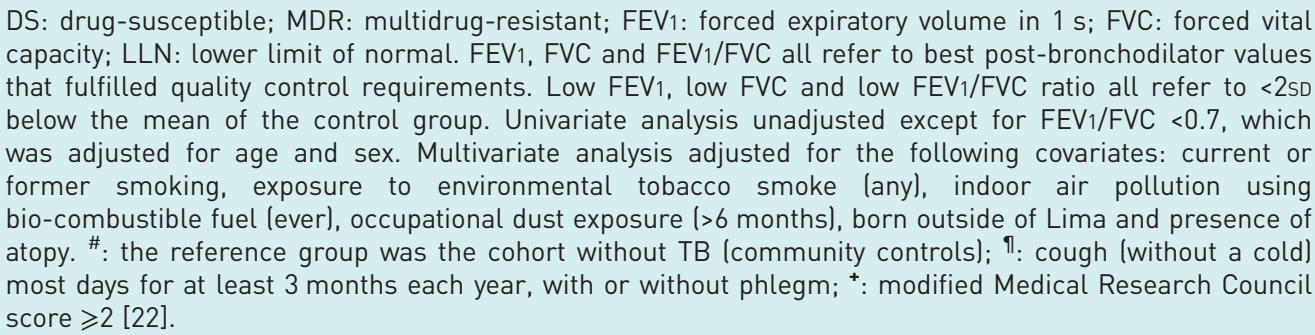 } \\
\hline
\end{tabular}

Our findings of minimal lung function impairment among patients who have been successfully treated for DS-TB is good news. We suspect that the ready availability of public healthcare facilities in Lima that offer free access to smear microscopy and chest radiography for those with respiratory symptoms, such as productive cough, may have contributed to these good respiratory outcomes by minimising diagnostic delay and concomitant lung damage. In cases with sputum smear-positive pulmonary TB, potent first-line treatment with isoniazid, rifampicin, ethambutol and pyrazinamide is commenced at the time of diagnosis [30] by a decentralised health centre. Nevertheless, the observed reduction in the FEV1/FVC ratio may be a signal that, while most patients treated for DS-TB have no functional lung disease, some do develop airflow obstruction.

As respiratory symptoms were common among all participants but did not differ between groups, spirometry should be considered in the medical assessment of any person with risk factors for airflow obstruction [31], including successfully treated TB. Although "milder", in terms of deviation from normal, this reduction in spirometric ratio may still be clinically significant. In a recent systematic review by HOESEIN et al. [32], there was an increased mortality as well as a greater number of hospitalisations among the people with a reduced $\mathrm{FEV}_{1} \mathrm{FVC}$ ratio, but preserved lung volumes, compared with those without airflow obstruction. This remains important from a public health perspective as the (DS-TB) cohort is young (median age 30 years) and would not usually be considered at risk for chronic airflow obstruction. 


\begin{tabular}{|c|c|c|c|c|}
\hline \multirow[t]{2}{*}{ Outcome } & \multicolumn{2}{|l|}{ DS-TB } & \multicolumn{2}{|l|}{ MDR-TB } \\
\hline & Absolute difference $(95 \% \mathrm{Cl})$ & p-value & Absolute difference $(95 \% \mathrm{Cl})$ & p-value \\
\hline \multicolumn{5}{|l|}{ FeNO ppb } \\
\hline Univariate & $1.12(0.98-1.28)$ & 0.11 & $0.93(0.74-1.16)$ & 0.53 \\
\hline Multivariate & $1.15(1.01-1.32)$ & 0.04 & $0.99(0.79-1.25)$ & 0.94 \\
\hline \multicolumn{5}{|l|}{ 6MWD m } \\
\hline Univariate & $4.13(-10.80-19.06)$ & 0.59 & $3.01(-21.79-27.80)$ & 0.81 \\
\hline Multivariate & $1.03(-14.14-16.20)$ & 0.89 & $-2.40(-27.46-22.65)$ & 0.85 \\
\hline \multicolumn{5}{|l|}{ FEV 1 L } \\
\hline Univariate & $-0.02(-0.17-0.13)$ & 0.82 & $-0.482(-0.727--0.237)$ & $<0.01$ \\
\hline Multivariate & $-0.07(-0.23-0.08)$ & 0.34 & $-0.431(-0.679--0.183)$ & $<0.01$ \\
\hline \multicolumn{5}{|l|}{ FVC L } \\
\hline Univariate & $0.04(-0.12-0.21)$ & 0.61 & $-0.426(-0.697--0.156)$ & $<0.01$ \\
\hline Multivariate & $-0.01(-0.18-0.15)$ & 0.88 & $-0.370(-0.644--0.097)$ & $<0.01$ \\
\hline \multicolumn{5}{|l|}{ FEV $/$ FVC } \\
\hline Univariate & $-0.02(-0.04-0.01)$ & 0.13 & $-0.059(-0.099--0.019)$ & $<0.01$ \\
\hline Multivariate & $-0.02(-0.05-0.00)$ & 0.10 & $-0.054(-0.096--0.012)$ & 0.01 \\
\hline \multicolumn{5}{|c|}{$\begin{array}{l}\text { DS: drug-susceptible; MDR: multidrug-resistant; FeNO: fraction of exhaled nitric oxide; 6MWD: 6-min walk } \\
\text { distance; FEV1: forced expiratory volume in } 1 \mathrm{~S} \text {; FVC: forced vital capacity. FEV1, FVC and FEV } 1 / F V C \text { all } \\
\text { refer to best post-bronchodilator values that fulfilled quality control requirements. Univariate analysis } \\
\text { unadjusted except for FEV1, FVC and FEV1/FVC, which were adjusted for age and sex, and FEV1 and FVC, } \\
\text { which were adjusted for height. Multivariate analysis adjusted for the following covariates: current or } \\
\text { former smoking, exposure to environmental tobacco smoke lany), indoor air pollution using } \\
\text { bio-combustible fuel (ever), occupational dust exposure ( }>6 \text { months), born outside of Lima and presence of } \\
\text { atopy. " : effect on FeNO expressed as ratio lexponent of the regression coefficient of group variable, with } \\
\text { logFeNO as the dependent variable). }\end{array}$} \\
\hline
\end{tabular}

Most would not have been included in COPD prevalence studies such as BOLD, PLATINO and CRONICAS $[4,5,33]$.

The scenario of rapid diagnosis and initiation of effective treatment is less likely to be the case for patients with sputum smear-negative or MDR-TB. Diagnostic delay and prolonged treatment with drugs that have low efficacy are the reality for most people with MDR-TB [34, 35]. We hypothesise that a delay in
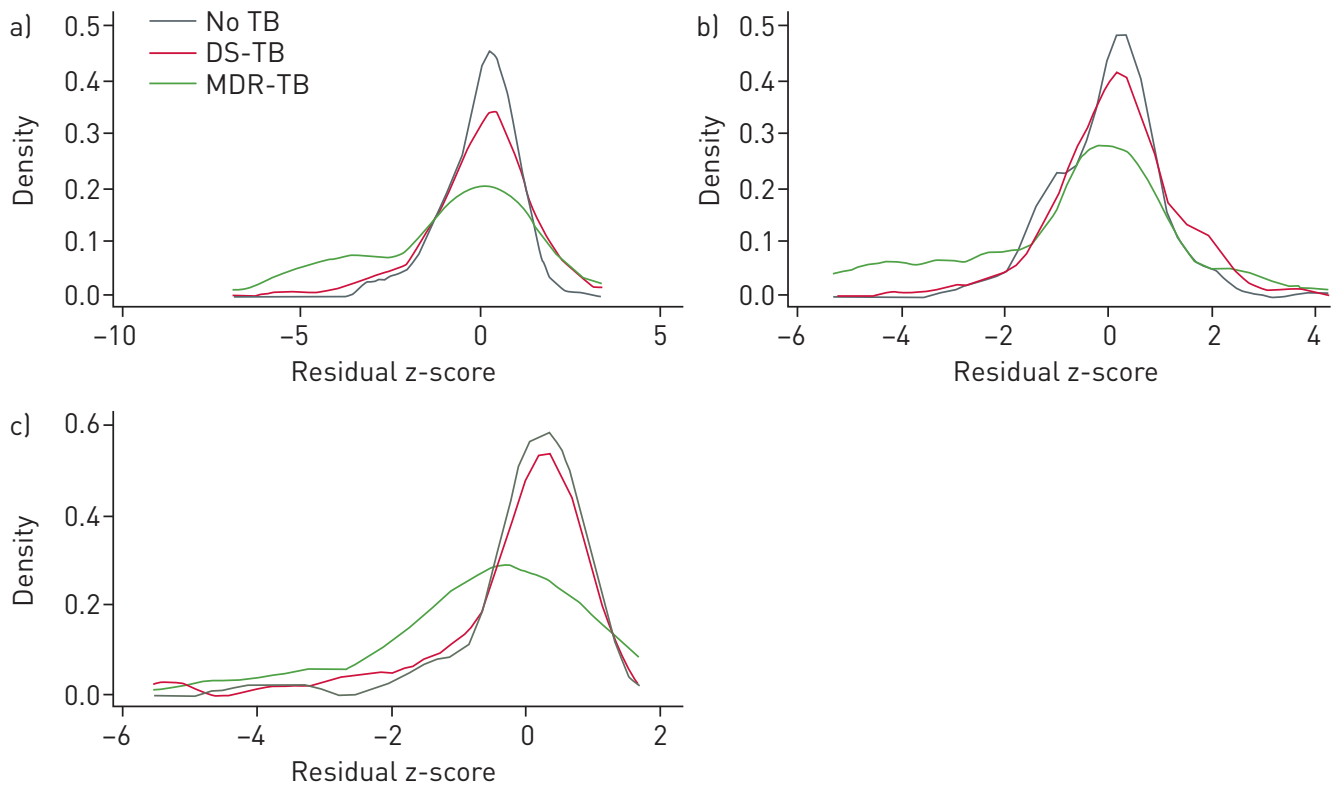

FIGURE 3 Density (frequency) distribution by group for the post-bronchodilator a) forced expiratory volume in $1 \mathrm{~s}$ (FEV 1 ), b) forced vital capacity (FVC) and c) FEV1/FVC ratio using height-, age- and sex-adjusted z-scores from the mean expected values of the control group. 
diagnosis and/or a lack of prompt and effective treatment is the main reason for the permanent loss of functional lung volume in patients with MDR-TB, rather than drug resistance per se. Importantly, all former TB patients were clinically cured and none had clinical or microbiological evidence of active disease at the time of evaluation. Thus, the presence of persistent mycobacterial disease was not thought to be the reason for the markedly impaired lung function observed in the MDR-TB cohort. Some patients with MDR-TB had lung volumes (FEV1 and FVC) like those observed in the cohort without TB, but an important subset had markedly reduced lung volumes. This subset may include those with the most delayed diagnosis and/or the most ineffective therapy, but we were unable to verify this. The diagnosis of MDR-TB has historically been difficult with limited access to drug susceptibility testing. Lima has a highly functional laboratory service, but cultures and drug susceptibility testing at the regional laboratory still take several weeks to perform [36]. The initiation of an appropriate MDR regimen also requires the approval of a central panel that meets on a weekly basis, which may further delay the initiation of appropriate treatment. However, the risk of more extensive lung involvement and greater functional impairment [37] needs to be balanced against the need to ensure optimal and appropriate therapy. The WHO now recommends the use of rapid (molecular) diagnostic tests for rifampicin resistance to enable prompt identification of, and initiation of treatment for, MDR-TB to reduce the risk of ongoing transmission [38]. This intervention may also be important in reducing the burden of chronic lung disease sequelae.

Although our study was not specifically designed to assess chronic airflow obstruction among the general population, the proportion of the control group with chronic airflow obstruction $(7.1 \%$ using a fixed ratio definition) is consistent with recently published data from the CRONICAS study in Peru in which participants were all aged $>35$ years $(6.2 \%$, also defined as a post-bronchodilator FEV1/FVC $<70 \%)$ [33]. Interestingly, the CRONICAS investigators also demonstrated an association between a history of pulmonary TB, as well as bio-combustible fuel use, and COPD, but the study was not designed to assess factors such as drug resistance or time since TB treatment. Our findings demonstrate that for this cohort of TB patients from Lima with confirmed successful microbiological treatment outcomes, the difference in lung function between TB patients and controls is not explained by the traditional environmental exposures of cigarette smoking or bio-combustible fuel use. TB can, however, result in irreversible impairment to functional volumes (FEV1 and FVC) as well as airway obstruction (reduced FEV1/FVC ratio), particularly when MDR disease is present. The early recognition of chronic airflow obstruction enables practitioners to instigate treatments that may alter disease progression and limit further loss of lung function [39].

Study limitations included the potential for nonresponder bias, due to the lower than expected participation rates. We sought to reduce this by maximising our efforts to recruit all selected patients. The MDR-TB cohort contained fewer patients and participation rates were lower, but this was mainly due to some cohort members being uncontactable. The proportion of potential participants who declined to participate after being successfully contacted was similar in the DS- and MDR-TB subcohorts. While we were unable to collect data from those who failed to participate, previous cohort studies have shown that participants with a lower education level and worse lung function were more likely to be nonparticipants at follow-up [40]. Therefore, the likelihood of bias towards the detection of functional lung impairment in study participants compared with nonparticipants seems low, although this cannot be excluded with confidence. The number of participants in the MDR-TB cohort was less than the original power calculation of 50, but the observed effect size was greater than that used for the sample size estimate and our findings were statistically significant. Study strengths included the cohort study design, which enrolled patients with confirmed successful treatment outcomes. This eliminated the risk of recall bias that was a feature of many previous studies. The population-based random sampling of control participants from the same communities as the $\mathrm{TB}$ cases provided robust internal controls to assess the impact of $\mathrm{TB}$ on respiratory outcomes, independent of other environmental, genetic and lifestyle factors. In addition, the risk of misclassification bias was considered low, since all participants undertook standardised post-bronchodilator spirometry with rigorous attention to quality control.

In conclusion, the early diagnosis and effective treatment of DS-TB resulted in minimal lung function impairment. Despite eventual cure, likely delays in the diagnosis and initiation of effective treatment in cases with MDR-TB probably explain the chronic airflow obstruction and lung volume loss observed in these patients. These findings support the urgent need to rapidly diagnose and treat all TB cases, particularly those with $\mathrm{DR}-\mathrm{TB}$, to limit ongoing TB transmission and reduce the burden of young adults in low- and middle-income countries with non-smoking-related chronic respiratory disease.

\section{Acknowledgements}

The authors wish to thank all the subjects that volunteered their time to participate in this study, as well as the dedicated study personnel that included Veronica Suarez, Bertha Diaz, Javier Silva, Orlando Hilario and Erika Villanueva (Socios En Salud Sucursal, Lima, Peru). 
Author contributions: A.L. Byrne had full access to all of the data in the study, and takes responsibility for the integrity of the data and the accuracy of the data analysis. A.L. Byrne, B.J. Marais, C.D. Mitnick, G.B. Marks, L. Lecca, C. Contreras, F. Garcia and Y. Yauri were responsible for the conception and design of the study. F.L. Garden, G.B. Marks, A.L. Byrne, C.D. Mitnick and B.J. Marais analysed and interpreted the data. A.L. Byrne, B.J. Marais, G.B. Marais, C.D. Mitnick, F.L. Garden, L. Lecca, C. Contreras and F. Garcia drafted the manuscript.

\section{References}

1 Burney PG, Patel J, Newson R, et al. Global and regional trends in COPD mortality, 1990-2010. Eur Respir J 2015; 45: 1239-1247.

2 Salvi SS, Barnes PJ. Chronic obstructive pulmonary disease in non-smokers. Lancet 2009; 374: 733-743.

3 Postma DS, Bush A, Van den Berge M. Risk factors and early origins of chronic obstructive pulmonary disease. Lancet 2015; 385: 899-909.

4 Buist AS, McBurnie MA, Vollmer WM, et al. International variation in the prevalence of COPD (the BOLD Study): a population-based prevalence study. Lancet 2007; 370: 741-750.

5 Menezes AM, Perez-Padilla R, Jardim JR, et al. Chronic obstructive pulmonary disease in five Latin American cities (the PLATINO study): a prevalence study. Lancet 2005; 366: 1875-1881.

6 Gauderman WJ, Vora H, McConnell R, et al. Effects of exposure to traffic on lung development from 10 to 18 years of age: a cohort study. Lancet 2007; 369: 571-577.

7 Mannino DM, Buist S. Global burden of COPD: risk factors, prevalence, and future trends. Lancet 2007; 370: 765-773.

8 WHO. Global Tuberculosis Report 2016. Geneva, World Health Organization, 2016.

9 Marais BJ. The global tuberculosis situation and the inexorable rise of drug-resistant disease. Adv Drug Deliv Rev 2016; 102: 3-9.

10 Singla R, Sarin R, Khalid UK, et al. Seven-year DOTS-Plus pilot experience in India: results, constraints and issues. Int J Tuberc Lung Dis 2009; 13: 976-981.

11 Hnizdo E, Singh T, Churchyard G. Chronic pulmonary function impairment caused by initial and recurrent pulmonary tuberculosis following treatment. Thorax 2000; 55: 32-38.

12 Jung KH, Kim SJ, Shin C, et al. The considerable, often neglected, impact of pulmonary tuberculosis on the prevalence of COPD. Am J Respir Crit Care Med 2008; 178: 431.

13 Byrne A, Marais B, Mitnick CD, et al. Risk factors for and origins of COPD. Lancet 2015; 385: 1723-1724.

14 Byrne AL, Marais BJ, Mitnick CD, et al. Tuberculosis and chronic respiratory disease: a systematic review. Int J Infect Dis 2015; 32: 138-146.

15 Lee C-H, Lee M-C, Lin H-H, et al. Pulmonary tuberculosis and delay in anti-tuberculous treatment are important risk factors for chronic obstructive pulmonary disease. PLoS One 2012; 7: e37978.

16 Cox HS, Morrow M, Detschmann PW. Long term efficacy of DOTS regimens for tuberculosis: systematic review. BMJ 2008; 336: 484-487.

17 The PLoS Medicine Editors. Observational studies: getting clear about transparency. PLoS Med 2014; 11: e1001711.

18 WHO. Training for Mid-Level Managers: The EPI Coverage Survey. Geneva, World Health Organization, 1991.

19 Miller MR, Crapo R, Hankinson J, et al. General considerations for lung function testing. Eur Respir J 2005; 26: $153-161$.

20 Quanjer PH, Stanojevic S, Cole TJ, et al. The ERS Global Lung Function Initiative. Eur Respir J 2012; 40: $1324-1343$.

21 Rennard SI, Vestbo J, Agustí A. What is chronic obstructive pulmonary disease anyway? Continua, categories, cut points, and moving beyond spirometry. Am J Respir Crit Care Med 2013; 187: 1036-1037.

22 Launois C, Barbe C, Bertin E, et al. The modified Medical Research Council scale for the assessment of dyspnea in daily living in obesity: a pilot study. BMC Pulm Med 2012; 12: 61.

23 Anno H, Tomashefski JF. Studies on the impairment of respiratory function in pulmonary tuberculosis. Am Rev Tuberc 1955; 71: 333-348.

24 Fox WE. Studies on the treatment of tuberculosis undertaken by the British Medical Research Council Tuberculosis Units (and relevant subsequent publications). Int J Tuberc Lung Dis 1999; S231-S279.

25 Jasmer RM, Seaman CB, Gonzalez LC, et al. Tuberculosis treatment outcomes. Am J Respir Crit Care Med 2004 170: $561-566$.

26 Jindani A, Doré CJ, Mitchison DA. Bactericidal and sterilizing activities of antituberculosis drugs during the first 14 days. Am J Respir Crit Care Med 2003; 167: 1348-1354.

27 Maguire GP, Anstey N, Ardian M, et al. Pulmonary tuberculosis, impaired lung function, disability and quality of life in a high-burden setting. Int J Tuberc Lung Dis 2009; 13: 1500-1507.

28 Pasipanodya JG, Miller TL, Vecino M, et al. Pulmonary impairment after tuberculosis. Chest 2007; 131: $1817-1824$.

29 Plit ML, Anderson R, Van Rensburg CE, et al. Influence of antimicrobial chemotherapy on spirometric parameters and pro-inflammatory indices in severe pulmonary tuberculosis. Eur Respir J 1998; 12: 351-356.

30 Minesterio de Salud. Impacto socioeconomico de la tuberculosis en el Perú 2010. [Socioeconomic impact of tuberculosis in Peru 2010.] http://bvs.minsa.gob.pe/local/minsa/1820.pdf Date last accessed: June 5, 2016.

31 Csikesz NG, Gartman EJ. New developments in the assessment of COPD: early diagnosis is key. Int J Chron Obstruct Pulmon Dis 2014; 9: 277-286.

32 Hoesein FA, Zanen P, Lammers JW. Lower limit of normal or FEV $1 / F V C<0.70$ in diagnosing COPD: an evidence-based review. Respir Med 2011; 105: 907-915.

33 Jaganath D, Miranda JJ, Gilman RH, et al. Prevalence of chronic obstructive pulmonary disease and variation in risk factors across four geographically diverse resource-limited settings in Peru. Respir Res 2015; 16: 2-9.

34 Greenaway C, Menzies D, Fanning A, et al. Delay in diagnosis among hospitalized patients with active tuberculosis - predictors and outcomes. Am J Respir Crit Care Med 2002; 165: 927-933.

35 Chung-Delgado K, Guillen-Bravo S, Revilla-Montag A, et al. Mortality among MDR-TB cases: comparison with drug-susceptible tuberculosis and associated factors. PLoS One 2015; 10: e0119332. 
36 Shin SS, Yagui M, Ascencios L, et al. Scale-up of multidrug-resistant tuberculosis laboratory services, Peru. Emerg Infect Dis 2008; 14: 701-708.

37 Schaaf HS, Shean K, Donald PR. Culture confirmed multidrug resistant tuberculosis: diagnostic delay, clinical features, and outcome. Arch Dis Child 2003; 88: 1106-1111.

38 Falzon D, Jaramillo E, Schunemann HJ, et al. WHO guidelines for the programmatic management of drug-resistant tuberculosis: 2011 update. Eur Respir J 2011; 38: 516-528.

39 Csikesz NG, Gartman EJ. New developments in the assessment of COPD: early diagnosis is key. Int J Chron Obstruct Pulm Dis 2014; 9: 277-286.

40 Mannino DM, Reichert MM, Davis KJ. Lung function decline and outcomes in an adult population. Am J Respir Crit Care Med 2006; 173: 985-990. 